\title{
On a Quaternionic Analogue of the Cross-Ratio
}

\author{
Ewain Gwynne*and Matvei Libine ${ }^{\dagger}$
}

December 7, 2011

\begin{abstract}
In this article we study an exact analogue of the cross-ratio for the algebra of quaternions $\mathbb{H}$ and use it to derive several interesting properties of quaternionic fractional linear transformations. In particular, we show that there exists a fractional linear transformation $T$ on $\mathbb{H}$ mapping four distinct quaternions $q_{1}, q_{2}, q_{3}$ and $q_{4}$ into $q_{1}^{\prime}, q_{2}^{\prime}, q_{3}^{\prime}$ and $q_{4}^{\prime}$ respectively if and only if the quadruples $\left(q_{1}, q_{2}, q_{3}, q_{4}\right)$ and $\left(q_{1}^{\prime}, q_{2}^{\prime}, q_{3}^{\prime}, q_{4}^{\prime}\right)$ have the same cross-ratio. If such a fractional linear transformation $T$ exists it is never unique. However, we prove that a fractional linear transformation on $\mathbb{H}$ is uniquely determined by specifying its values at five points in general position. We also prove some properties of the cross-ratio including criteria for four quaternions to lie on a single circle (or a line) and for five quaternions to lie on a single 2-sphere (or a 2-plane). As an application of the cross-ratio, we prove that fractional linear transformations on $\mathbb{H}$ map spheres (or affine subspaces) of dimension 1, 2 and 3 into spheres (or affine subspaces) of the same dimension.
\end{abstract}

Keywords: quaternions, cross-ratio, fractional linear transformations, conformal transformations, Möbius transformations, Möbius geometry.

\section{Introduction}

In this article we study an exact analogue of the cross-ratio for the algebra of quaternions $\mathbb{H}$ and use it to derive several interesting properties of quaternionic fractional linear transformations. (Note that some authors prefer to call fractional linear transformations "Möbius transformations".) Recall that, if $z_{1}, z_{2}, z_{3}, z_{4} \in \mathbb{C}$ are distinct complex numbers, then their cross-ratio is

$$
R_{\mathbb{C}}\left(z_{1}, z_{2}, z_{3}, z_{4}\right)=\frac{\left(z_{4}-z_{1}\right)\left(z_{2}-z_{3}\right)}{\left(z_{2}-z_{1}\right)\left(z_{4}-z_{3}\right)} .
$$

The cross-ratio has many important properties including invariance under fractional linear transformations:

$$
R_{\mathbb{C}}\left(z_{1}, z_{2}, z_{3}, z_{4}\right)=R_{\mathbb{C}}\left(T\left(z_{1}\right), T\left(z_{2}\right), T\left(z_{3}\right), T\left(z_{4}\right)\right)
$$

for all fractional linear transformations $T$ on $\mathbb{C}$. Another way to characterize the cross-ratio is as follows. Let $T$ be the unique fractional linear transformation sending $z_{1}, z_{2}$ and $z_{3}$ into 0,1 and $\infty$ respectively, then $R_{\mathbb{C}}\left(z_{1}, z_{2}, z_{3}, z_{4}\right)=T\left(z_{4}\right)$. The cross-ratio can be used to determine if given four distinct points $z_{1}, z_{2}, z_{3}, z_{4} \in \mathbb{C}$ lie on a circle or a straight line. This happens if and only if $R_{\mathbb{C}}\left(z_{1}, z_{2}, z_{3}, z_{4}\right)$ is real. As a consequence, one immediately obtains that a fractional linear transformation maps circles and lines into circles and lines. These properties are discussed in detail in most complex analysis textbooks including, for example, [A].

\footnotetext{
${ }^{*}$ Undergraduate student at Northwestern University, Evanston, IL 60208

${ }^{\dagger}$ Department of Mathematics, Indiana University, Rawles Hall, 831 East 3rd St, Bloomington, IN 47405
} 
In Section 2 we introduce our notations and list some basic facts about quaternions.

In Section 3 we state the definition of the quaternionic cross-ratio (Definition 7); it is essentially the same as the one introduced in $[\mathrm{BP}],[\mathrm{HJHP}]$ and $[\mathrm{HJ}]$. We prove that there exists a fractional linear transformation $T$ on $\mathbb{H}$ mapping four distinct points $q_{1}, q_{2}, q_{3}$ and $q_{4}$ into $q_{1}^{\prime}, q_{2}^{\prime}, q_{3}^{\prime}$ and $q_{4}^{\prime}$ respectively if and only if the quadruples $\left(q_{1}, q_{2}, q_{3}, q_{4}\right)$ and $\left(q_{1}^{\prime}, q_{2}^{\prime}, q_{3}^{\prime}, q_{4}^{\prime}\right)$ have cross-ratios with the same real parts and norms (Theorem 8 ). The fact that the fractional linear transformations preserve the real part and the norm of the quaternionic cross-ratio was proved in [HJ] by a different method. The invariants of fractional linear transformations discussed in Remark 9 were suggested to us by Igor Frenkel (see also Proposition 3 in [C]). We also note that a fractional linear transformation on $\mathbb{H}$ is never determined uniquely by specifying its values at four points.

In Section 4 we derive various properties of the cross-ratio and fractional linear transformations on $\mathbb{H}$. First, we fix the images of three points in $\mathbb{H}$ and show that the set of all possible images of a fourth point under a fractional linear transformation is either a 2-sphere, a 2-plane or a single point (Proposition 10). As a consequence of this result, we show that fractional linear transformations on $\mathbb{H}$ map spheres (or affine subspaces) of dimension 1,2 and 3 into spheres (or affine subspaces) of the same dimension (Theorem 11). Theorem 11 was originally proved in $[\mathrm{BG}]$, but their proof did not use the quaternionic cross-ratio. Then we show that four quaternions $q_{1}, q_{2}, q_{3}, q_{4}$ lie on a single circle (or a line) if and only if their cross-ratio is real (Proposition [12). The last property is not new, see, for example, [BP], [HJHP], [HJ], [BG].

In Section 5 we give a necessary and sufficient condition in terms of cross-ratios for the existence of a fractional linear transformation with prescribed values at five different points (Proposition 13). We prove that such a fractional linear transformation is uniquely determined if these five points do not lie on a single 2-sphere or a 2-plane (Proposition 14). Then we give a criterion for five quaternions to lie on a single 2-sphere (or a 2-plane) (Lemma 15). Finally, we fix the images of four points in $\mathbb{H}$ and show that the set of all possible images of a fifth point under a fractional linear transformation is either a circle, a line or a single point (Proposition 16). To the best of authors knowledge, the results of this section are new.

Finally, we comment that other properties of the quaternionic cross-ratio are discussed in the book [HJ]. Moreover, there is a Clifford algebra analogue of the cross-ratio (for example, see $[\mathrm{C}],[\mathrm{BHJ}],[\mathrm{HJ}]$ ), and it would be interesting to determine if the results of our article extend to Clifford algebras. For example, Proposition 1 in [C] is a Clifford algebra analogue of Proposition 12. We expect most results stated in our paper to have such an extension.

This paper was written as a part of REU (research experiences for undergraduates) project at Indiana University during Summer 2011. We would like to thank Professors Kevin Pilgrim and Bruce Solomon, and the NSF for providing the organization and funding for the REU program that made this project possible. This REU program was supported by the NSF grant DMS-0851852. The second author was supported by the NSF grant DMS-0904612.

\section{Preliminaries}

In this section we introduce our notations and list basic facts about quaternions that we will use. There are many texts providing elementary introductions to quaternions including, for example, $[\mathrm{BFLPP}$ and $[\mathrm{HJ}]$. Recall that the quaternions $\mathbb{H}$ form an algebra over $\mathbb{R}$ generated by the units $1, i, j, k$. The multiplicative structure is determined by the rules

$$
\begin{gathered}
1 q=q 1=q, \quad \forall q \in \mathbb{H}, \\
i j=-j i, \quad i k=-k i, \quad j k=-k j, \\
i^{2}=j^{2}=k^{2}=i j k=-1,
\end{gathered}
$$


and the fact that $\mathbb{H}$ is a division ring. We write elements $q \in \mathbb{H}$ as

$$
q=t+i x+j y+k z, \quad t, x, y, z \in \mathbb{R}
$$

and use notations for

$$
\begin{array}{ll}
\text { quaternionic conjugate: } & \bar{q}=t-i x-j y-k z, \\
\text { norm: } & |q|=\sqrt{q \bar{q}}=\sqrt{\bar{q}}=\sqrt{t^{2}+x^{2}+y^{2}+z^{2}} \quad \in \mathbb{R}, \\
\text { real part: } & \operatorname{Re} q=(q+\bar{q}) / 2=t \quad \in \mathbb{R}, \\
\text { imaginary part: } & \operatorname{Im} q=(q-\bar{q}) / 2=i x+j y+k z \quad \in \mathbb{H}, \\
\text { non-zero quaternions: } & \mathbb{H}^{\times}=\mathbb{H} \backslash\{0\} .
\end{array}
$$

Then we have

$$
\begin{aligned}
\overline{q_{1} q_{2}} & =\bar{q}_{2} \cdot \bar{q}_{1}, \\
\left|q_{1} q_{2}\right| & =\left|q_{1}\right| \cdot\left|q_{2}\right|, \\
\operatorname{Re}\left(q_{1} q_{2}\right) & =\operatorname{Re}\left(q_{2} q_{1}\right), \\
q^{-1} & =\bar{q} /|q|^{2} .
\end{aligned}
$$

Occasionally, it is convenient to use a matrix realization of quaternions. We realize $\mathbb{H}$ as a subalgebra of the algebra of $2 \times 2$ complex matrices by identifying the units $1, i, j, k \in \mathbb{H}$ with

$$
1 \leftrightarrow\left(\begin{array}{ll}
1 & 0 \\
0 & 1
\end{array}\right), \quad i \leftrightarrow\left(\begin{array}{cc}
0 & -i \\
-i & 0
\end{array}\right), \quad j \leftrightarrow\left(\begin{array}{cc}
0 & -1 \\
1 & 0
\end{array}\right), \quad k \rightsquigarrow\left(\begin{array}{cc}
-i & 0 \\
0 & i
\end{array}\right) .
$$

Thus

$$
q=t+i x+j y+k z \quad \leadsto \quad\left(\begin{array}{cc}
t-i z & -y-i x \\
y-i x & t+i z
\end{array}\right),
$$

and we get a matrix realization of quaternions:

$$
\mathbb{H} \simeq\left\{\left(\begin{array}{cc}
a & b \\
-\bar{b} & \bar{a}
\end{array}\right) \in \mathfrak{g l}(2, \mathbb{C}) ; a, b \in \mathbb{C}\right\}
$$

Under this identification, $|q|^{2}$ and $2 \operatorname{Re} q$ are respectively the determinant and trace of the corresponding matrix, and the unit sphere in $\mathbb{H}$ gets identified with $S U(2)$ in $G L(2, \mathbb{C})$ :

$$
S^{3}=\{q \in \mathbb{H} ;|q|=1\} \simeq\left\{\left(\begin{array}{cc}
a & b \\
-\bar{b} & \bar{a}
\end{array}\right) \in G L(2, \mathbb{C}) ; a, b \in \mathbb{C}, \operatorname{det}\left(\begin{array}{cc}
a & b \\
-\bar{b} & \bar{a}
\end{array}\right)=1\right\}=S U(2) .
$$

Each non-zero quaternion $a \in \mathbb{H}^{\times}$induces a transformation Conj $_{a}$ on $\mathbb{H}$ :

$$
\operatorname{Conj}_{a}: q \mapsto a q a^{-1}, \quad \forall q \in \mathbb{H} .
$$

The transformation $\operatorname{Conj}_{a}$ preserves the real parts and norms, hence determines a rotation in the 3-dimensional space consisting of purely imaginary quaternions, i.e. an element of $O(3)$. Because $\mathbb{H}^{\times}$is connected, $\operatorname{Conj}_{a} \in S O(3)$ and we obtain a map Conj : $\mathbb{H}^{\times} \rightarrow S O(3), a \mapsto$ Conj $_{a}$. Since Conj $\mathrm{j}_{a}$ is the identity transformation whenever $a \in \mathbb{R}^{\times}=\mathbb{R} \backslash\{0\}$, Conj descends to a map

$$
\mathbb{H}^{\times} / \mathbb{R}^{\times}=S U(2) /\{ \pm I d\} \rightarrow S O(3) .
$$

It is well-known that this map is an analytic isomorphism. (See, for example, $[\mathrm{H}$, Example II in Chapter V, §2.) Note that if $\operatorname{Im} a \neq 0$, then the axis of the rotation $\operatorname{Conj}_{a}$ is the line passing through $\operatorname{Im} a$. These two lemmas follow immediately from the above discussion: 
Lemma 1. Let $q, q^{\prime} \in \mathbb{H}$, then $q^{\prime}=a q a^{-1}$ for some $a \in \mathbb{H}^{\times}$if and only if $|q|=\left|q^{\prime}\right|$ and $\operatorname{Re} q=\operatorname{Re} q^{\prime}$.

Lemma 2. Two quaternions $q_{1}, q_{2} \in \mathbb{H}$ commute with each other if and only if one of $\operatorname{Im} q_{1}$, $\operatorname{Im} q_{2}$ is a real multiple of the other.

Let $G L(2, \mathbb{H})$ be the group consisting of invertible $2 \times 2$ matrices with entries in $\mathbb{H}$. It acts on $\widehat{\mathbb{H}}=\mathbb{H} \cup\{\infty\}$ by fractional linear (or Möbius) transformations:

$$
\pi(\gamma): q \mapsto(a q+b)(c q+d)^{-1}, \quad \text { where } \gamma=\left(\begin{array}{ll}
a & b \\
c & d
\end{array}\right) \in G L(2, \mathbb{H})
$$

First we show that there always exists a fractional linear transformation sending any three distinct points $q_{1}, q_{2}, q_{3}$ in $\widehat{\mathbb{H}}$ into 0,1 and $\infty$ respectively.

Lemma 3. Given any three distinct points $q_{1}, q_{2}, q_{3} \in \widehat{\mathbb{H}}$, there exists a fractional linear transformation $T_{q_{1}, q_{2}, q_{3}}$ such that $T_{q_{1}, q_{2}, q_{3}}\left(q_{1}\right)=0, T_{q_{1}, q_{2}, q_{3}}\left(q_{2}\right)=1$ and $T_{q_{1}, q_{2}, q_{3}}\left(q_{3}\right)=\infty$.

Proof. Applying a fractional linear transformation if necessary, we can assume that none of $q_{1}, q_{2}, q_{3}$ is $\infty$. Let

$$
T_{q_{1}, q_{2}, q_{3}}(q)=\left(q_{2}-q_{1}\right)^{-1}\left(q-q_{1}\right)\left(q-q_{3}\right)^{-1}\left(q_{2}-q_{3}\right) .
$$

Then $T_{q_{1}, q_{2}, q_{3}}$ maps $q_{1}$ to $0, q_{2}$ to $1, q_{3}$ to $\infty$, and $T_{q_{1}, q_{2}, q_{3}}=\pi(\gamma)$, where

$$
\gamma=\left(\begin{array}{cc}
\left(q_{2}-q_{1}\right)^{-1} & 0 \\
0 & \left(q_{2}-q_{3}\right)^{-1}
\end{array}\right)\left(\begin{array}{cc}
1 & -q_{1} \\
1 & -q_{3}
\end{array}\right) \in G L(2, \mathbb{H})
$$

We will often use this lemma to reduce the general case when $q_{1}, q_{2}, q_{3}$ are three distinct points in $\widehat{\mathbb{H}}$ to the case when $q_{1}=0, q_{2}=1, q_{3}=\infty$.

Corollary 4. Given any three distinct points $q_{1}, q_{2}, q_{3} \in \widehat{\mathbb{H}}$ and another triple of distinct points $q_{1}^{\prime}, q_{2}^{\prime}, q_{3}^{\prime} \in \widehat{\mathbb{H}}$, there exists a fractional linear transformation $T$ such that $T\left(q_{n}\right)=q_{n}^{\prime}, n=1,2,3$.

Proof. Setting $T=\left(T_{q_{1}^{\prime}, q_{2}^{\prime}, q_{3}^{\prime}}\right)^{-1} \circ T_{q_{1}, q_{2}, q_{3}}$ gives the desired transformation.

The following lemma implies that the fractional linear transformation in the above corollary is never unique.

Lemma 5. A fractional linear transformation $T$ maps 0 to 0,1 to 1 , and $\infty$ to $\infty$ if and only if $T$ is of the form $T(q)=a q a^{-1}$ for some $a \in \mathbb{H}^{\times}$.

Proof. Write $T(q)=(a q+b)(c q+d)^{-1}$ for some $a, b, c, d \in \mathbb{H}$. The condition that $T(0)=0$ implies that $b=0$; the condition that $T(\infty)=\infty$ implies that $c=0$; the condition that $T(1)=1$ implies that $a+b=c+d$, and hence that $a=d$. Thus, $T(q)=a q a^{-1}$, as desired.

We finish this section by restating Lemma 10 from $[\mathrm{FL}]$ :

Lemma 6. Let $q_{1}, q_{2} \in \mathbb{H}, \gamma=\left(\begin{array}{ll}a & b \\ c & d\end{array}\right) \in G L(2, \mathbb{H})$. Write $\gamma^{-1}=\left(\begin{array}{ll}a^{\prime} & b^{\prime} \\ c^{\prime} & d^{\prime}\end{array}\right), \tilde{q}_{1}=\left(a q_{1}+\right.$ b) $\left(c q_{1}+d\right)^{-1}$ and $\tilde{q}_{2}=\left(a q_{2}+b\right)\left(c q_{2}+d\right)^{-1}$. Then

$$
\begin{aligned}
\left(\tilde{q}_{1}-\tilde{q}_{2}\right) & =\left(a^{\prime}-q_{2} c^{\prime}\right)^{-1} \cdot\left(q_{1}-q_{2}\right) \cdot\left(c q_{1}+d\right)^{-1} \\
& =\left(a^{\prime}-q_{1} c^{\prime}\right)^{-1} \cdot\left(q_{1}-q_{2}\right) \cdot\left(c q_{2}+d\right)^{-1} .
\end{aligned}
$$




\section{Quaternionic Cross-Ratio}

In this section we introduce a quaternionic analogue of the cross-ratio and prove some of its properties.

Definition 7. Given four distinct points $q_{1}, q_{2}, q_{3}, q_{4} \in \widehat{\mathbb{H}}$, we define their cross-ratio to be

$$
Q\left(q_{1}, q_{2}, q_{3}, q_{4}\right)=\left(q_{2}-q_{1}\right)^{-1}\left(q_{4}-q_{1}\right)\left(q_{4}-q_{3}\right)^{-1}\left(q_{2}-q_{3}\right) \quad \in \mathbb{H} .
$$

(If one of the $q_{n}=\infty$, the cross-ratio is defined by letting this $q_{n} \rightarrow \infty$ and taking limits.)

Setting $q_{1}=0, q_{2}=1$ and letting $q_{3} \rightarrow \infty$ we get:

$$
Q(0,1, \infty, q)=q
$$

It will be convenient to introduce a notation

$$
R_{\mathbb{H}}\left(q_{1}, q_{2}, q_{3}, q_{4}\right)=\left(\left|Q\left(q_{1}, q_{2}, q_{3}, q_{4}\right)\right|, \operatorname{Re} Q\left(q_{1}, q_{2}, q_{3}, q_{4}\right)\right) \quad \in \mathbb{R}^{2} .
$$

Comparing the definition of $R_{\mathbb{H}}$ with the complex case (11), note that the magnitude of a complex number together with its real part determine that complex number up to complex conjugation, but this is not the case for quaternions.

This definition originally appeared in $[\mathrm{BP}]$, where the cross-ratio was defined for purely imaginary quaternions only, then in [HJHP] and [HJ] it was extended to all quaternions. Note that some authors define the quaternionic cross-ratio as

$$
\operatorname{Re} Q\left(q_{1}, q_{2}, q_{3}, q_{4}\right) \pm i\left|\operatorname{Im} Q\left(q_{1}, q_{2}, q_{3}, q_{4}\right)\right| \quad \in \mathbb{C}
$$

This is a complex number defined up to conjugation which uniquely determines $R_{\mathbb{H}}\left(q_{1}, q_{2}, q_{3}, q_{4}\right)$ and in turn can be recovered from $R_{\mathbb{H}}\left(q_{1}, q_{2}, q_{3}, q_{4}\right)$.

Perhaps, the most important property of the quaternionic cross-ratio is that the quantity $R_{\mathbb{H}}\left(q_{1}, q_{2}, q_{3}, q_{4}\right)$ stays invariant under fractional linear transformations. This property is a part of the following theorem.

Theorem 8. Given four distinct points $q_{1}, q_{2}, q_{3}, q_{4} \in \widehat{\mathbb{H}}$ and another quadruple of distinct points $q_{1}^{\prime}, q_{2}^{\prime}, q_{3}^{\prime}, q_{4}^{\prime} \in \widehat{\mathbb{H}}$, there exists a fractional linear transformation $T$ such that $T\left(q_{n}\right)=q_{n}^{\prime}$, $n=1,2,3,4$, if and only if

$$
R_{\mathbb{H}}\left(q_{1}, q_{2}, q_{3}, q_{4}\right)=R_{\mathbb{H}}\left(q_{1}^{\prime}, q_{2}^{\prime}, q_{3}^{\prime}, q_{4}^{\prime}\right)
$$

Proof. First we show that $R_{\mathbb{H}}\left(q_{1}, q_{2}, q_{3}, q_{4}\right)$ is invariant under fractional linear transformations. Pick any $\gamma=\left(\begin{array}{ll}a & b \\ c & d\end{array}\right) \in G L(2, \mathbb{H})$, let $T(q)=(a q+b)(c q+d)^{-1}$ be the corresponding fractional linear transformation and write $\gamma^{-1}=\left(\begin{array}{ll}a^{\prime} & b^{\prime} \\ c^{\prime} & d^{\prime}\end{array}\right)$. By Lemma 6,

$$
\begin{aligned}
& Q\left(T\left(q_{1}\right), T\left(q_{2}\right), T\left(q_{3}\right), T\left(q_{4}\right)\right) \\
&=\left(c q_{2}+d\right)\left(q_{2}-q_{1}\right)^{-1}\left(q_{4}-q_{1}\right)\left(q_{4}-q_{3}\right)^{-1}\left(q_{2}-q_{3}\right)\left(c q_{2}+d\right)^{-1} \\
&=\left(c q_{2}+d\right) Q\left(q_{1}, q_{2}, q_{3}, q_{4}\right)\left(c q_{2}+d\right)^{-1}
\end{aligned}
$$

and it follows from Lemma 1 that

$$
R_{\mathbb{H}}\left(T\left(q_{1}\right), T\left(q_{2}\right), T\left(q_{3}\right), T\left(q_{4}\right)\right)=R_{\mathbb{H}}\left(q_{1}, q_{2}, q_{3}, q_{4}\right) .
$$


Conversely, suppose that $R_{\mathbb{H}}\left(q_{1}, q_{2}, q_{3}, q_{4}\right)=R_{\mathbb{H}}\left(q_{1}^{\prime}, q_{2}^{\prime}, q_{3}^{\prime}, q_{4}^{\prime}\right)$. By Lemma 3 , without loss of generality we can assume that $q_{1}=q_{1}^{\prime}=0, q_{2}=q_{2}^{\prime}=1, q_{3}=q_{3}^{\prime}=\infty$. Then by (2) we have

$$
\left|q_{4}\right|=\left|q_{4}^{\prime}\right|, \quad \operatorname{Re} q_{4}=\operatorname{Re} q_{4}^{\prime} .
$$

It follows from Lemmas 1 and 5 that there exists a fractional linear transformation $T: q \mapsto$ $a q a^{-1}$ such that $T(0)=0, T(1)=1, T(\infty)=\infty$ and $T(q)=q^{\prime}$.

Remark 9. One can generate more invariants of fractional linear transformations as follows. For an even number of points $q_{1}, \ldots, q_{2 n} \in \mathbb{H}$, define

$$
\tilde{Q}\left(q_{1}, \ldots, q_{2 n}\right)=\left(q_{1}-q_{2}\right)\left(q_{2}-q_{3}\right)^{-1}\left(q_{3}-q_{4}\right)\left(q_{4}-q_{5}\right)^{-1} \ldots\left(q_{2 n-1}-q_{2 n}\right)\left(q_{2 n}-q_{1}\right)^{-1} .
$$

Then it follows from Lemma 9 that

$$
\left|\tilde{Q}\left(q_{1}, \ldots, q_{2 n}\right)\right|=\left|\tilde{Q}\left(T\left(q_{1}\right), \ldots, T\left(q_{2 n}\right)\right)\right|, \quad \operatorname{Re} \tilde{Q}\left(q_{1}, \ldots, q_{2 n}\right)=\operatorname{Re} \tilde{Q}\left(T\left(q_{1}\right), \ldots, T\left(q_{2 n}\right)\right)
$$

for all fractional linear transformations $T$. One can even set some of these points equal to each other. For example, choose $n=3$ and set $q_{3}=q_{6}$, then the norm and the real part of

$$
\tilde{Q}\left(q_{1}, q_{2}, q_{3}, q_{4}, q_{5}\right)=\left(q_{1}-q_{2}\right)\left(q_{2}-q_{3}\right)^{-1}\left(q_{3}-q_{4}\right)\left(q_{4}-q_{5}\right)^{-1}\left(q_{5}-q_{3}\right)\left(q_{3}-q_{1}\right)^{-1} .
$$

remain unchanged under fractional linear transformations.

Note that specifying the values of a fractional linear transformation $T$ at four points never determines the transformation uniquely. This is because any four points in $\mathbb{H}$ lie on a 2 -sphere or a 2-plane. Any 2-sphere or 2-plane in $\mathbb{H}$ can be transformed by a fractional linear transformation into the unit imaginary sphere

$$
\Theta=\{q \in \mathbb{H} ;|q|=1, \operatorname{Re} q=0\} .
$$

So, without loss of generality we can assume that $T$ fixes four points on $\Theta$. One can show that the set of all fractional linear transformations fixing $\Theta$ consists of the rotation matrices:

$$
\{\gamma \in G L(2, \mathbb{H}) ; \pi(\gamma)(q)=q \forall q \in \Theta\}=\left\{\gamma_{\theta}=\left(\begin{array}{cc}
\cos \theta & -\sin \theta \\
\sin \theta & \cos \theta
\end{array}\right) \in G L(2, \mathbb{H}) ; \theta \in \mathbb{R}\right\} .
$$

Finally, composing $T$ with $\pi\left(\gamma_{\theta}\right)$ results in new fractional linear transformations that have the same values at the selected four points. In Proposition 14 we will give conditions that determine a fractional linear transformation uniquely.

\section{Properties of the Quaternionic Cross-Ratio}

In this section we discuss some properties of the quaternionic cross-ratio and fractional linear transformations on $\mathbb{H}$. First we fix the images of three points in $\mathbb{H}$ and geometrically characterize all possible images of a fourth point under fractional linear transformations.

Proposition 10. Let $q_{1}, q_{2}, q_{3}, q_{4} \in \widehat{\mathbb{H}}$ be four distinct points, and let $q_{1}^{\prime}, q_{2}^{\prime}, q_{3}^{\prime} \in \widehat{\mathbb{H}}$ be three distinct points. Then the set

$$
S=\left\{\pi(\gamma)\left(q_{4}\right) ; \gamma \in G L(2, \mathbb{H}), \pi(\gamma)\left(q_{n}\right)=q_{n}^{\prime}, n=1,2,3\right\} \quad \subset \widehat{\mathbb{H}}
$$

is either a 2-sphere, a 2-plane or a single point. The degenerate case when this set is a point happens if and only if $Q\left(q_{1}, q_{2}, q_{3}, q_{4}\right)$ is real. 
Proof. To simplify notations, we write $Q$ for $Q\left(q_{1}, q_{2}, q_{3}, q_{4}\right)$. By Theorem $8, s \in S$ if and only if

$$
\begin{aligned}
\left|\left(q_{2}^{\prime}-q_{1}^{\prime}\right)^{-1}\left(s-q_{1}^{\prime}\right)\left(s-q_{3}^{\prime}\right)^{-1}\left(q_{2}^{\prime}-q_{3}^{\prime}\right)\right| & =|Q| \quad \text { and } \\
\operatorname{Re}\left(\left(q_{2}^{\prime}-q_{1}^{\prime}\right)^{-1}\left(s-q_{1}^{\prime}\right)\left(s-q_{3}^{\prime}\right)^{-1}\left(q_{2}^{\prime}-q_{3}^{\prime}\right)\right) & =\operatorname{Re} Q .
\end{aligned}
$$

Expanding (5) and using the properties of the norm gives

$$
|Q|^{2}=\frac{\left|q_{2}^{\prime}-q_{3}^{\prime}\right|^{2} \cdot\left|s-q_{1}^{\prime}\right|^{2}}{\left|q_{2}^{\prime}-q_{1}^{\prime}\right|^{2} \cdot\left|s-q_{3}^{\prime}\right|^{2}} \quad \Longleftrightarrow \quad\left|s-q_{1}^{\prime}\right|^{2}=|Q|^{2} \frac{\left|q_{2}^{\prime}-q_{1}^{\prime}\right|^{2} \cdot\left|s-q_{3}^{\prime}\right|^{2}}{\left|q_{2}^{\prime}-q_{3}^{\prime}\right|^{2}} .
$$

Write

$$
\begin{aligned}
s & =t+x i+y j+z k, \\
q_{1}^{\prime} & =a_{0}+a_{1} i+a_{2} j+a_{3} k, \\
q_{3}^{\prime} & =b_{0}+b_{1} i+b_{2} j+b_{3} k, \\
A & =|Q|^{2} \frac{\left|q_{2}^{\prime}-q_{1}^{\prime}\right|^{2}}{\left|q_{2}^{\prime}-q_{3}^{\prime}\right|^{2}} .
\end{aligned}
$$

Then the above equation becomes

$\left(t-a_{0}\right)^{2}+\left(x-a_{1}\right)^{2}+\left(y-a_{2}\right)^{2}+\left(z-a_{3}\right)^{2}=A\left(\left(t-b_{0}\right)^{2}+\left(x-b_{1}\right)^{2}+\left(y-b_{2}\right)^{2}+\left(z-b_{3}\right)^{2}\right)$.

If $A=1$, this is the equation of a 3-plane. Otherwise, expanding and completing the square for each variable gives an equation of the form

$$
\left(t-c_{0}\right)^{2}+\left(x-c_{1}\right)^{2}+\left(y-c_{2}\right)^{2}+\left(z-c_{3}\right)^{2}=B
$$

for some $c_{0}, c_{1}, c_{2}, c_{3}, B \in \mathbb{R}$. Since this equation has at least one solution, $B \geq 0$ and this is the equation of either a 3 -sphere in $\mathbb{H}$ or a single point.

Consider now the condition (6). We can rewrite it as follows:

$$
\operatorname{Re}\left(\left(q_{2}^{\prime}-q_{3}^{\prime}\right)\left(q_{2}^{\prime}-q_{1}^{\prime}\right)^{-1}\left(s-q_{1}^{\prime}\right)\left(s-q_{3}^{\prime}\right)^{-1}\right)=\operatorname{Re} Q .
$$

Write

$$
\begin{aligned}
\alpha=\left(q_{2}^{\prime}-q_{3}^{\prime}\right)\left(q_{2}^{\prime}-q_{1}^{\prime}\right)^{-1} & =\alpha_{0}+\alpha_{1} i+\alpha_{2} j+\alpha_{3} k, \\
\beta=q_{3}^{\prime}-q_{1}^{\prime} & =\beta_{0}+\beta_{1} i+\beta_{2} j+\beta_{3} k, \\
u=s-q_{3}^{\prime} & =t+x i+y j+z k .
\end{aligned}
$$

Then (6) becomes

$$
\operatorname{Re} Q=\operatorname{Re}\left(\alpha(u+\beta) u^{-1}\right)=\operatorname{Re} \alpha+|u|^{-2} \operatorname{Re}(\alpha \beta \bar{u})=\alpha_{0}+|u|^{-2}\left(\delta_{0} t+\delta_{1} x+\delta_{2} y+\delta_{3} z\right)
$$

for some real numbers $\delta_{0}, \delta_{1}, \delta_{2}, \delta_{3}$ that can be expressed in terms of $\alpha_{n}$ 's and $\beta_{m}$ 's. Multiplying through by $|u|^{2}$ gives

$$
\left(\operatorname{Re} Q-\alpha_{0}\right)\left(t^{2}+x^{2}+y^{2}+z^{2}\right)-\left(\delta_{0} t+\delta_{1} x+\delta_{2} y+\delta_{3} z\right)=0 .
$$

Once again, if $\operatorname{Re} Q-\alpha_{0}=0$, this is the equation for a 3-plane. Otherwise, completing the square for each variable gives

$$
\left(t-d_{0}\right)^{2}+\left(x-d_{1}\right)^{2}+\left(y-d_{2}\right)^{2}+\left(z-d_{3}\right)^{2}=D .
$$


Since this equation has at least one solution, $D \geq 0$ and this is the equation of either a 3 -sphere in $\mathbb{H}$ or a single point.

Thus, the set $S$ can be realized as an intersection of two sets, each of which is either a 3 -sphere, a 3-plane or a point. Since $S$ is non-empty, this implies that $S$ is either a 3sphere, a 3-plane, a 2-sphere, a 2-plane or a point. Since fractional linear transformations are diffeomorphisms, they preserve the dimensions of submanifolds. Hence, to pin down the dimension of $S$, we can use Lemma 3 and assume without loss of generality that $q_{1}=q_{1}^{\prime}=0$, $q_{2}=q_{2}^{\prime}=1, q_{3}=q_{3}^{\prime}=\infty$. Then by Lemmas 1 and [5, the set $S$ is either 2-dimensional or a single point, and the latter case happens if and only if $a Q\left(q_{1}, q_{2}, q_{3}, q_{4}\right) a^{-1}=Q\left(q_{1}, q_{2}, q_{3}, q_{4}\right)$ for all $a \in \mathbb{H}^{\times}$, i.e. if and only if $Q\left(q_{1}, q_{2}, q_{3}, q_{4}\right)$ is real.

Recall that fractional linear transformations over $\mathbb{C}$ map circles and straight lines into circles and straight lines. As a consequence of the above proposition we obtain:

Theorem 11. Fractional linear transformations on $\widehat{\mathbb{H}}$ send circles and lines into circles and lines, 2-spheres and 2-planes into 2-spheres and 2-planes, 3-spheres and 3-planes into 3-spheres and 3-planes.

Proof. Consider first a 2-sphere or a 2-plane $S$. Note that any 2-sphere (respectively 2-plane) in $\mathbb{H}$ can be transformed into any other 2-sphere (respectively 2-plane) by a transformation of the type $q \mapsto a q+b, a, b \in \mathbb{H}$. Hence we can assume that our set $S$ can be realized as (4) for some choice of $q_{1}, q_{2}, q_{3}, q_{4} \in \widehat{\mathbb{H}}$. Now, let $T$ be any fractional linear transformation. Then

$$
T(S)=\left\{\pi(\gamma)\left(q_{4}\right) ; \gamma \in G L(2, \mathbb{H}), \pi(\gamma)\left(q_{n}\right)=T\left(q_{n}^{\prime}\right), n=1,2,3\right\}
$$

which by the above proposition is either a 2-sphere or a 2-plane.

Now, let $C \subset \widehat{\mathbb{H}}$ be a circle or a line. Any circle or line can be expressed as an intersection $C=S_{1} \cap S_{2}$, where $S_{1}$ and $S_{2}$ are 2-spheres or 2-planes. Then $T(C)=T\left(S_{1}\right) \cap T\left(S_{2}\right)$ is the intersection of 2-spheres and/or 2-planes, so is also either a circle or a line.

Finally, let $R$ be a 3-sphere or a 3-plane. Applying a linear transformation $q \mapsto a q+b$ as above and using the characterization of the set of points satisfying $\left|Q\left(q_{1}, q_{2}, q_{3}, q\right)\right|=$ const given in the proof of Proposition 10, we can realize $R$ as

$$
R=\left\{q \in \mathbb{H} ;\left|Q\left(q_{1}, q_{2}, q_{3}, q\right)\right|=N\right\}
$$

for some choice of $q_{1}, q_{2}, q_{3} \in \widehat{\mathbb{H}}$ and $N \in(0, \infty)$. Then

$$
T(R)=\left\{q \in \mathbb{H} ;\left|Q\left(T\left(q_{1}\right), T\left(q_{2}\right), T\left(q_{3}\right), q\right)\right|=N\right\},
$$

which is a 3-sphere or a 3-plane as well.

Theorem 11] was originally proved in [BG], but their proof did not use the quaternionic crossratio. Instead, they checked that 3-spheres and 3-planes get mapped into 3-spheres and 3-planes by the generators of the group of fractional linear transformations $\{\pi(\gamma) ; \gamma \in G L(2, \mathbb{H})\}$, namely the translations, rotations, dilations and the inversion $q \mapsto q^{-1}$. Then they realized 2-spheres, 2-planes, circles and lines as finite intersections of 3-spheres and 3-planes.

Recall that, over complex numbers $\mathbb{C}$, the cross-ratio (11) is real if and only if the four points $z_{1}, z_{2}, z_{3}, z_{4} \in \mathbb{C}$ lie on a single circle or a single line. This result also carries over to $\mathbb{H}$ :

Proposition 12. Let $q_{1}, q_{2}, q_{3}, q_{4} \in \widehat{\mathbb{H}}$. Then $Q\left(q_{1}, q_{2}, q_{3}, q_{4}\right)$ is real if and only if $q_{1}, q_{2}, q_{3}, q_{4}$ lie on a single circle or a single line. 
Proof. Let $T$ denote the fractional linear transformation $T_{q_{1}, q_{2}, q_{3}}$ from Lemma 3 . Then, by Theorem 11, $q_{1}, q_{2}, q_{3}, q_{4}$ lie on a single circle or a line if and only if the points $0,1, \infty, T\left(q_{4}\right)$ do, i.e. if and only if $T\left(q_{4}\right)$ is real. But, $T\left(q_{4}\right)$ is exactly the quaternion $Q\left(q_{1}, q_{2}, q_{3}, q_{4}\right)$.

Recall from Proposition 10 that the set $S$ defined by (4) degenerates into a single point if and only if the cross-ratio $Q\left(q_{1}, q_{2}, q_{3}, q_{4}\right)$ is real. Now we know that this happens if and only if the points $q_{1}, q_{2}, q_{3}, q_{4}$ lie on single circle or a single line.

\section{Five Points and the Unique Determination of Fractional Lin- ear Transformations}

As it was explained after Remark 9, specifying values of a fractional linear transformation at four points never determines that transformation uniquely. We shall give a necessary and sufficient condition for the existence of a fractional linear transformation with prescribed values at five different points and discuss the uniqueness of such a transformation.

Proposition 13. Let $q_{1}, q_{2}, q_{3}, q_{4}, q_{5} \in \widehat{\mathbb{H}}$ be five distinct points, and let $q_{1}^{\prime}, q_{2}^{\prime}, q_{3}^{\prime}, q_{4}^{\prime}, q_{5}^{\prime} \in \widehat{\mathbb{H}}$ be another collection of five distinct points. Then there exists a fractional linear transformation $T$ such that $T\left(q_{n}\right)=q_{n}^{\prime}, n=1,2,3,4,5$, if and only if there exists an $a \in \mathbb{H}^{\times}$such that

$$
Q\left(q_{1}^{\prime}, q_{2}^{\prime}, q_{3}^{\prime}, q_{4}^{\prime}\right)=a Q\left(q_{1}, q_{2}, q_{3}, q_{4}\right) a^{-1} \quad \text { and } \quad Q\left(q_{1}^{\prime}, q_{2}^{\prime}, q_{3}^{\prime}, q_{5}^{\prime}\right)=a Q\left(q_{1}, q_{2}, q_{3}, q_{5}\right) a^{-1} .
$$

Proof. For simplicity write

$$
Q_{4}=Q\left(q_{1}, q_{2}, q_{3}, q_{4}\right), \quad Q_{4}^{\prime}=Q\left(q_{1}^{\prime}, q_{2}^{\prime}, q_{3}^{\prime}, q_{4}^{\prime}\right), \quad Q_{5}=Q\left(q_{1}, q_{2}, q_{3}, q_{5}\right), \quad Q_{5}^{\prime}=Q\left(q_{1}^{\prime}, q_{2}^{\prime}, q_{3}^{\prime}, q_{5}^{\prime}\right)
$$

By (3), if $T$ is a fractional linear transformation on $\widehat{\mathbb{H}}$, replacing $q_{n}$ with $T\left(q_{n}\right), n=1,2,3,4,5$, results in conjugating $Q_{4}$ and $Q_{5}$ by the same quaternion. Thus, by Lemma 3, we can assume without loss of generality that $q_{1}=q_{1}^{\prime}=0, q_{2}=q_{2}^{\prime}=1, q_{3}=q_{3}^{\prime}=\infty$. Then, by (2), we have $Q_{4}=q_{4}, Q_{4}^{\prime}=q_{4}^{\prime}, Q_{5}=q_{5}$ and $Q_{5}^{\prime}=q_{5}^{\prime}$. On the other hand, by Lemma 5 , any transformation $T$ such that $T\left(q_{n}\right)=q_{n}^{\prime}, n=1,2,3$, has to be of the form $q \mapsto a q a^{-1}$ for some $a \in \mathbb{H}^{\times}$. This proves that there exists a fractional linear transformation $T$ such that $T\left(q_{n}\right)=q_{n}^{\prime}, n=1,2,3,4,5$, if and only if there exists an $a \in \mathbb{H}^{\times}$such that $Q_{4}^{\prime}=a Q_{4} a^{-1}$ and $Q_{5}^{\prime}=a Q_{5} a^{-1}$.

In light of the discussion preceding Lemma 1, we can restate Proposition 13 as follows. Given five distinct points $q_{1}, q_{2}, q_{3}, q_{4}, q_{5} \in \widehat{\mathbb{H}}$ and five more distinct points $q_{1}^{\prime}, q_{2}^{\prime}, q_{3}^{\prime}, q_{4}^{\prime}, q_{5}^{\prime} \in \widehat{\mathbb{H}}$, there exists a fractional linear transformation $T$ such that $T\left(q_{n}\right)=q_{n}^{\prime}, n=1,2,3,4,5$, if and only if

1. $\left|Q\left(q_{1}, q_{2}, q_{3}, q_{4}\right)\right|=\left|Q\left(q_{1}^{\prime}, q_{2}^{\prime}, q_{3}^{\prime}, q_{4}^{\prime}\right)\right|$ and $\left|Q\left(q_{1}, q_{2}, q_{3}, q_{5}\right)\right|=\left|Q\left(q_{1}^{\prime}, q_{2}^{\prime}, q_{3}^{\prime}, q_{5}^{\prime}\right)\right|$;

2. $\operatorname{Re} Q\left(q_{1}, q_{2}, q_{3}, q_{4}\right)=\operatorname{Re} Q\left(q_{1}^{\prime}, q_{2}^{\prime}, q_{3}^{\prime}, q_{4}^{\prime}\right)$ and $\operatorname{Re} Q\left(q_{1}, q_{2}, q_{3}, q_{5}\right)=\operatorname{Re} Q\left(q_{1}^{\prime}, q_{2}^{\prime}, q_{3}^{\prime}, q_{5}^{\prime}\right) ;$

3. There is a single rotation of the 3 -space consisting of imaginary quaternions which takes

$$
\operatorname{Im} Q\left(q_{1}, q_{2}, q_{3}, q_{4}\right) \mapsto \operatorname{Im} Q\left(q_{1}^{\prime}, q_{2}^{\prime}, q_{3}^{\prime}, q_{4}^{\prime}\right) \quad \text { and } \quad \operatorname{Im} Q\left(q_{1}, q_{2}, q_{3}, q_{5}\right) \mapsto \operatorname{Im} Q\left(q_{1}^{\prime}, q_{2}^{\prime}, q_{3}^{\prime}, q_{5}^{\prime}\right)
$$

In general, if $p_{1}, p_{2}, p_{1}^{\prime}, p_{2}^{\prime}$ are points in $\mathbb{R}^{3}$, then there exists a rotation $\mathcal{R} \in S O(3)$ such that $\mathcal{R}\left(p_{1}\right)=p_{1}^{\prime}$ and $\mathcal{R}\left(p_{2}\right)=p_{2}^{\prime}$ if and only if $\left|p_{1}\right|=\left|p_{1}^{\prime}\right|,\left|p_{2}\right|=\left|p_{2}^{\prime}\right|$ and $\left|p_{1}-p_{2}\right|=\left|p_{1}^{\prime}-p_{2}^{\prime}\right|$. Thus, in the presence of the first two conditions, the last condition is satisfied if and only if

$$
3^{\prime} .\left|Q\left(q_{1}, q_{2}, q_{3}, q_{4}\right)-Q\left(q_{1}, q_{2}, q_{3}, q_{5}\right)\right|=\left|Q\left(q_{1}^{\prime}, q_{2}^{\prime}, q_{3}^{\prime}, q_{4}^{\prime}\right)-Q\left(q_{1}^{\prime}, q_{2}^{\prime}, q_{3}^{\prime}, q_{5}^{\prime}\right)\right| .
$$


Next we address the question of uniqueness of fractional linear transformations on $\widehat{\mathbb{H}}$.

Proposition 14. Let $q_{1}, q_{2}, q_{3}, q_{4}, q_{5} \in \widehat{\mathbb{H}}$ be five distinct points not lying on a single 2-sphere or 2-plane. Then any fractional linear transformation $T$ is uniquely determined by its values at these points, $T\left(q_{n}\right), n=1,2,3,4,5$.

Proof. By Lemma 3 and Theorem 11, without loss of generality we can assume that $q_{1}=$ $T\left(q_{1}\right)=0, q_{2}=T\left(q_{2}\right)=1$ and $q_{3}=T\left(q_{3}\right)=\infty$. Then by, Lemma 5, $T$ has to be of the form $q \mapsto a q a^{-1}$ for some $a \in \mathbb{H}^{\times}$. Hence $T$ preserves the real parts and is effectively a rotation of the imaginary 3 -space. Since the points $q_{1}, q_{2}, q_{3}, q_{4}, q_{5}$ do not lie on a single 2 -plane, $\operatorname{Im} q_{4} \neq 0$, $\operatorname{Im} q_{5} \neq 0$ and $\operatorname{Im} q_{5}$ is not a real multiple of $\operatorname{Im} q_{4}$. Therefore, $\operatorname{Im} q_{4}$ and $\operatorname{Im} q_{5}$ do not lie on a single line through the origin, and the rotation $T$ is uniquely determined by its values on $q_{4}$ and $q_{5}$.

To determine if five points lie on the same 2-sphere or a 2-plane we can use the cross-ratio:

Lemma 15. Five different points $q_{1}, q_{2}, q_{3}, q_{4}, q_{5} \in \widetilde{\mathbb{H}}$ lie on a single 2-sphere or a 2-plane if and only if $Q\left(q_{1}, q_{2}, q_{3}, q_{4}\right)$ and $Q\left(q_{1}, q_{2}, q_{3}, q_{5}\right)$ commute with each other.

Proof. As in the proof of Proposition [13, let $Q_{4}=Q\left(q_{1}, q_{2}, q_{3}, q_{4}\right)$ and $Q_{5}=Q\left(q_{1}, q_{2}, q_{3}, q_{5}\right)$. By (3), if $T$ is a fractional linear transformation on $\widehat{\mathbb{H}}$, replacing $q_{n}$ with $T\left(q_{n}\right), n=1,2,3,4,5$, results in conjugating $Q_{4}$ and $Q_{5}$ by the same quaternion. Thus by Lemma 3 and Theorem 11 , we can assume without loss of generality that $q_{1}=0, q_{2}=1, q_{3}=\infty$. By (2), we have $Q_{4}=q_{4}$ and $Q_{5}=q_{5}$. Then the points $q_{1}, q_{2}, q_{3}, q_{4}, q_{5}$ lie on a single 2-plane if and only if one of $\operatorname{Im} Q_{4}$, $\operatorname{Im} Q_{5}$ is a real multiple of the other. By Lemma 2, this is equivalent to $Q_{4}$ and $Q_{5}$ commuting with each other.

Finally, we fix the images of four points in $\mathbb{H}$ and characterize all possible images of a fifth point under fractional linear transformations, just like we did in Proposition 10 with four points.

Proposition 16. Let $q_{1}, q_{2}, q_{3}, q_{4}, q_{5} \in \widehat{\mathbb{H}}$ be five distinct points, and let $q_{1}^{\prime}, q_{2}^{\prime}, q_{3}^{\prime}, q_{4}^{\prime} \in \widehat{\mathbb{H}}$ be four distinct points. Assume that

$$
R_{\mathbb{H}}\left(q_{1}, q_{2}, q_{3}, q_{4}\right)=R_{\mathbb{H}}\left(q_{1}^{\prime}, q_{2}^{\prime}, q_{3}^{\prime}, q_{4}^{\prime}\right)
$$

and that $\operatorname{Im} Q\left(q_{1}, q_{2}, q_{3}, q_{4}\right) \neq 0$ (or, equivalently, that the points $q_{1}, q_{2}, q_{3}, q_{4}$ do not lie on a single circle or a line). Then the set

$$
C=\left\{\pi(\gamma)\left(q_{5}\right) ; \gamma \in G L(2, \mathbb{H}), \pi(\gamma)\left(q_{n}\right)=q_{n}^{\prime}, n=1,2,3,4\right\} \quad \subset \widehat{\mathbb{H}}
$$

is either a circle, a line or a single point. The degenerate case when this set is a point happens if and only if $Q\left(q_{1}, q_{2}, q_{3}, q_{4}\right)$ and $Q\left(q_{1}, q_{2}, q_{3}, q_{5}\right)$ commute with each other.

Proof. By Lemma 3 and Theorem[11, without loss of generality we can assume that $q_{1}=q_{1}^{\prime}=0$, $q_{2}=q_{2}^{\prime}=1, q_{3}=q_{3}^{\prime}=\infty$. Then, by (2),

$$
Q\left(q_{1}, q_{2}, q_{3}, q_{4}\right)=q_{4} \quad \text { and } \quad Q\left(q_{1}, q_{2}, q_{3}, q_{5}\right)=q_{5}
$$

by Lemma 5 ,

$$
C=\left\{a q_{5} a^{-1} ; a \in \mathbb{H}^{\times}, a q_{4} a^{-1}=q_{4}^{\prime}\right\} ;
$$

by Theorem 8 and assumption (7), the set $C$ is non-empty.

Geometrically, following the discussion preceding Lemma 1, we are essentially looking at the set of all $\mathcal{R}\left(\operatorname{Im} q_{5}\right)$, where $\mathcal{R} \in S O(3)$ runs over all rotations taking $\operatorname{Im} q_{4}$ into $\operatorname{Im} q_{4}^{\prime}$. But 
all such rotations have the form $\mathcal{R}^{\prime} \circ \mathcal{R}_{0}$, where $\mathcal{R}_{0} \in S O(3)$ is a fixed rotation such that $\mathcal{R}_{0}\left(\operatorname{Im} q_{4}\right)=\operatorname{Im} q_{4}^{\prime}$ and $\mathcal{R}^{\prime} \in S O(3)$ is a rotation about the line passing through $\operatorname{Im} q_{4}^{\prime}$. This proves that the set $C$ is either a circle, a line or a single point.

The case when $C$ is a point happens if and only if the rotation $\mathcal{R}_{0}$ takes $\operatorname{Im} q_{5}$ into a point lying on the line passing through $\operatorname{Im} q_{4}^{\prime}$. That happens if and only if $\operatorname{Im} q_{5}$ is a real multiple of $\operatorname{Im} q_{4}$, which, by Lemma 2, happens if and only if $Q\left(q_{1}, q_{2}, q_{3}, q_{4}\right)$ and $Q\left(q_{1}, q_{2}, q_{3}, q_{5}\right)$ commute.

\section{References}

[A] L. V. Ahlfors, Complex Analysis, 3rd edition, McGraw-Hill, New York, 1978.

[BG] C. Bisi, G. Gentili. Möbius transformations and the Poincaré distance in the quaternionic setting, Indiana Univ. Math. Journal 58 (2009), 2729-2764; also arXiv:0805.0357.

[BHJ] A. Bobenko, U. Hertrich-Jeromin, Orthogonal nets and Clifford algebras, in Proceedings of the Fifth Pacific Rim Geometry Conference, Tohoku Math. Publ., 2001, pp. $7-22$.

[BP] A. Bobenko, U. Pinkall, Discrete isothermic surfaces, J. reine angew, Math 475 (1996), 187-208.

[BFLPP] F. E. Burstall, D. Ferus, K. Leschke, F. Pedit, U. Pinkall, Conformal geometry of surfaces in $S^{4}$ and quaternions, Lecture Notes in Mathematics, 1772, Springer-Verlag, Berlin, 2002.

[C] J. Cieśliński, The cross ratio and Clifford algebras, Advances in Applied Clifford Algebras 7 no. 2 (1997), 133-139.

[FL] I. Frenkel, M. Libine, Quaternionic analysis, representation theory and physics, Advances in Math 218 (2008), 1806-1877; also arXiv:0711.2699.

[H] S. Helgason, Differential Geometry, Lie Groups, and Symmetric Spaces, AMS, Providence, 2001.

[HJ] U. Hertrich-Jeromin, Introduction to Möbius Differential Geometry, London Mathematical Society Lecture Note Series, 300, Cambridge University Press, Cambridge, 2003.

[HJHP] U. Hertrich-Jeromin, T. Hoffmann, U. Pinkall A discrete version of the Darboux transform for isometric surfaces, in A. Bobenko,R. Seiler (Eds.), Discrete integrable geometry and physics, Oxford University Press, 1999, pp. 59-81. 